\title{
Primary carcinoid tumors of the liver
}

\section{Gary Schwartz ${ }^{* 1}$, Agnes Colanta ${ }^{2}$, Harold Gaetz ${ }^{2}$, John Olichney ${ }^{3}$ and Fadi Attiyeh ${ }^{1}$}

Address: ${ }^{1}$ Department of Surgery, 1000 10th Avenue, Suite 2B, New York, NY, 10019, USA, 2Department of Pathology, St. Luke's-Roosevelt Hospital Center, 1000 10th Avenue, 1st Floor, New York, NY, 10019, USA and '3epartment of Hematology-Oncology, St. Luke's-Roosevelt Hospital Center, 350 West 58th Street, New York, NY, 10019, USA

Email: Gary Schwartz* - gsschwartz@gmail.com; Agnes Colanta - acolanta@chpnet.org; Harold Gaetz - hgaetz@chpnet.org; John Olichney - jolichney@chpnet.org; Fadi Attiyeh - fattiyeh@chpnet.org

* Corresponding author

Published: 27 August 2008

World Journal of Surgical Oncology 2008, 6:91 doi:10.1186/1477-78|9-6-9|

This article is available from: http://www.wjso.com/content/6/I/9I

(C) 2008 Schwartz et al; licensee BioMed Central Ltd.

This is an Open Access article distributed under the terms of the Creative Commons Attribution License (http://creativecommons.org/licenses/by/2.0), which permits unrestricted use, distribution, and reproduction in any medium, provided the original work is properly cited.
Received: 21 June 2008
Accepted: 27 August 2008

\begin{abstract}
Background: Primary carcinoid tumors of the liver are uncommon and rarely symptomatic. The diagnosis of primary hepatic etiology requires rigorous workup and continued surveillance to exclude a missed primary.

Case Presentation: We present a case of a 62-year-old female with a primary hepatic carcinoid tumor successfully resected, now with three years of disease-free follow-up. We present a review of the current literature regarding the diagnosis, pathology, management, and natural history of this disease entity.

Conclusion: Primary carcinoid tumors of the liver are rare, therefore classifying their nature as primary hepatic in nature requires extensive workup and prolonged follow-up. All neuroendocrine tumors have an inherent malignant potential that must be recognized. Management remains surgical resection, with several alternative options available for non-resectable tumors and severe symptoms. The risk of recurrence of primary hepatic carcinoid tumors after resection remains unknown.
\end{abstract}

\section{Background}

Although carcinoid tumors can be found throughout the body, 90\% occur within the gastrointestinal tract [1]. They preferentially metastasize to the liver and occasionally (< $10 \%$ ) cause the carcinoid syndrome by secretion of serotonin and its precursors, as well as other vasoactive substances [2]. Primary carcinoid tumors of the liver are exceedingly rare, with only about 60 cases reported in the current literature. Meticulous follow-up is necessary to rule out an occult extrahepatic malignancy with hepatic metastasis to confirm the primary nature of hepatic carcinoids.

\section{Case presentation}

EG is a 62-year-old female who presented with right upper quadrant abdominal pain, intermittent in timing and dull in nature, not related to oral intake and not associated with nausea or vomiting. Her past medical history included hypertension, irritable bowel syndrome, osteoarthritis, and a history of recurrent bilateral lower extremity deep venous thrombosis on Warfarin. On physical exam there were no abdominal scars, normal bowel sounds on auscultation, minimal right upper quadrant tenderness to palpation, no rebound tenderness or guarding, no hepatomegaly and a negative Murphy's sign. Her 
laboratory studies were significant for a GGT of $162 \mathrm{U} / \mathrm{L}$ (normal 5-80 U/L), with otherwise normal liver function tests. Tumor markers were negative, with an AFP of $3.1 \mathrm{ng} /$ $\mathrm{ml}$.

Diagnostic imaging included an abdominal ultrasound (Figure 1) which revealed a heterogeneous solid mass in the lateral segment of the left hepatic lobe measuring 6.3 $\times 5.3 \times 5.0 \mathrm{~cm}$. A CT scan with intravenous contrast was obtained which revealed a $4.9 \times 4.9 \mathrm{~cm}$ enhancing, poorly marginated mass in segment II of the liver, with no other intra-abdominal masses or lymphadenopathy (Figure 2).

A CT-guided biopsy was performed which yielded scant tissue with poorly cohesive cells arranged in papillae. PASD stain showed focal, small mucin droplets in some cells. Immunohistochemistry was positive for CEA and CK-7 and negative for calretinin, CDX-2, CK-20, Muc-2 and Muc-6. The limited sample was diagnosed as papillary adenocarcinoma, favoring metastasis, on the basis of morphology, special stain results and immunoprofile. However, a second panel of immunohistochemical stains for synaptophysin, CD56 and chromogranin were performed on the biopsy specimen. The tumor cells were negative for chromogranin but expressed synaptophysin and CD56, consistent with the immunoprofile of a neuroendocrine tumor (NET).

Further workup for a primary tumor or other metastatic sites included a negative CT scan of the chest, upper and lower gastrointestinal endoscopy, and a Technetium-99m

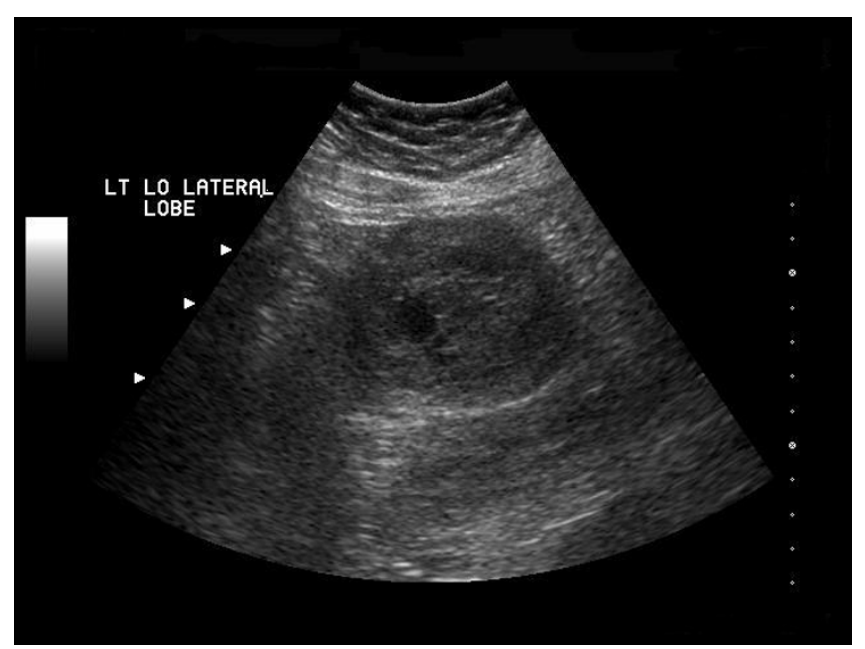

Figure I

Ultrasound of the abdomen; Ultrasound of the abdomen depicting a $6.3 \times 5.3 \times 5.0$ heterogenous solid mass in the lateral segment of the left lobe of the liver.

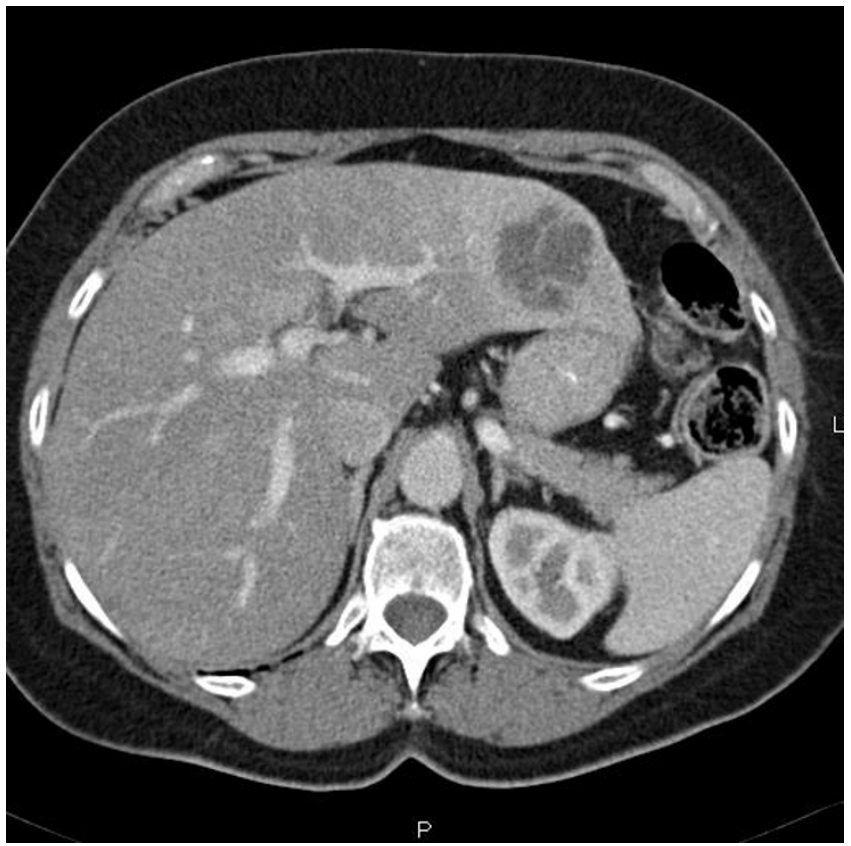

Figure 2

CT scan of the abdomen and pelvis; CT scan of the abdomen and pelvis with IV contrast demonstrates a $4.9 \times 4.9 \mathrm{~cm}$ enhancing, poorly marginated mass in segment II of the liver.

bone scan. The decision was made to resect the hepatic tumor.

An uncomplicated left lateral segmentectomy (II \& III) and cholecystectomy were performed. No peritoneal carcinomatosis was noted upon exploration. The postoperative course was uneventful and she was discharged home on the fourth postoperative day.

Grossly, the tumor measured $5.2 \times 5.0 \times 5.0 \mathrm{~cm}$ and had a tan gray, soft, fish-fleshy cut surface (Figure 3 ). Although there was a focal infiltrative edge, it was well-circumscribed and located $5.9 \mathrm{~cm}$ away from the resection margin. The tumor consisted of approximately $40 \%$ solid areas and $60 \%$ hemorrhagic and cystic degenerative areas. There were no satellite nodules. Surgical margins were negative for malignancy, including the left hepatic artery, vein, duct, gallbladder and portal lymph nodes.

Microscopically, the tumor consisted predominantly of solid sheets and organoid nests of uniform, intermediatesized, polyhedral cells (Figure 4A) in a vascular stroma. Other areas showed a trabecular arrangement of these cells with focal stromal hyalinization (Figure 4B); cystic areas were also present. Cytologically, the tumor cells had a moderate amount of eosinophilic cytoplasm with perinuclear eosinophilic inclusions and round to oval nuclei 


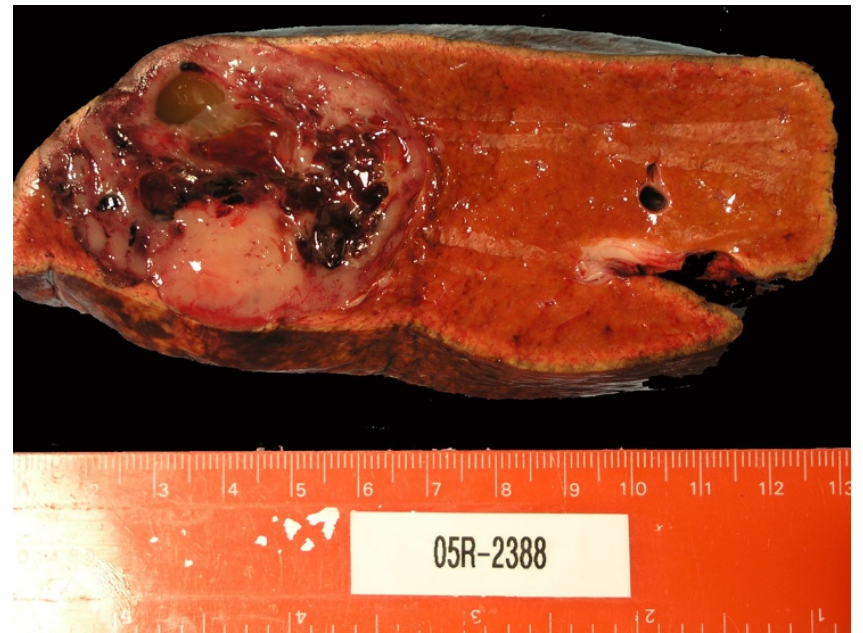

Figure 3

Gross image of the specimen; The specimen was measured at $5.2 \times 5.0 \times 5.0 \mathrm{~cm}$ and had a tan gray, soft, fish-fleshy cut surface.

with vesicular to finely granular chromatin. There were no areas of necrosis, and mitoses were infrequent.

Immunohistochemistry was consistent with the immunoprofile of the biopsy specimen, i.e. positive staining for synaptophysin (Figure 5A) and CD56 (Figure 5B) and negative staining for chromogranin. Additionally, there was immunoreactivity for epithelial markers CK-7, CAM

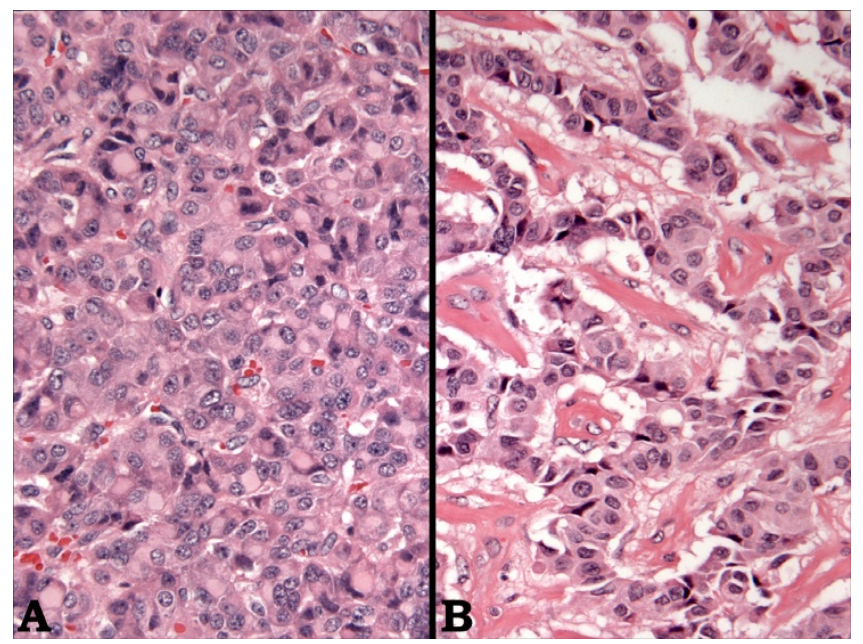

Figure 4

Microscopic image of the specimen; The tumor consisted of solid sheets and organoid nests of uniform, intermediate-sized, polyhedral cells in a vascular stroma (Image A) as well as areas of trabecular arrangement with focal stromal hyalinization (Image B).

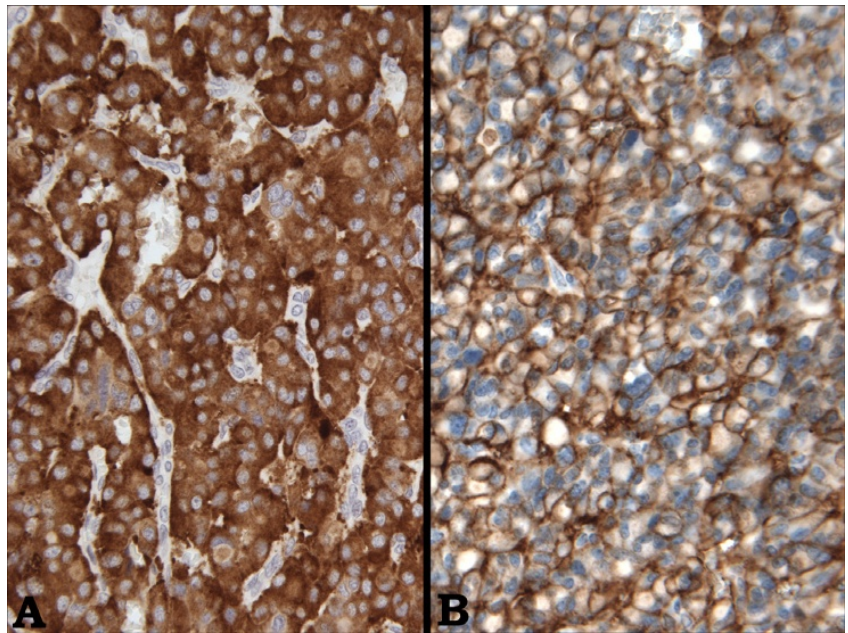

Figure 5

Immunohistochemistry of the resected specimen; Immunohistochemistry was positive for synaptophysin (Image A) and CD56 (Image B), consistent with a NET.

5.2 and pancytokeratin AE1/AE3. There was negative staining for HEPT, CA19.9 and TTF-1, thus ruling out hepatocellular carcinoma, metastatic carcinoma from the gastrointestinal tract and metastatic lung carcinoma, respectively. The histomorphologic features coupled with the immunohistochemical results supported the diagnosis of a carcinoid tumor/low grade NET.

Follow-up over the subsequent three years included CT scans of the abdomen at six month intervals. To date, no recurrent or metastatic disease has been identified. She remains symptom free and in good health.

\section{Discussion}

A total of sixty cases of primary hepatic carcinoid have been reported, with the largest series being eight patients [3], with long-term follow-up ranging from two to eleven years. Of the reported cases, there is a wide range of age at presentation and there does not seem to be gender predominance. There is no apparent association with cirrhosis or preexisting liver disease.

Primary hepatic carcinoid tumors may be an incidental finding or can present with severe symptoms including abdominal pain, jaundice, palpable right upper quadrant mass, carcinoid syndrome [4], carcinoid heart disease [5], and Cushing's Syndrome [6]. Less than $10 \%$ of gastrointestinal carcinoids present with the carcinoid syndrome and when the syndrome is present it is almost always associated with hepatic metastasis. Interestingly, the syndrome is rarely present in primary hepatic carcinoid tumors, with only two reported cases $[4,5]$. 
Imaging studies of any hepatic mass should begin with ultrasound and a triple-phase CT scan. One report supports the use of contrast-enhanced ultrasound, although there is limited experience with that modality [7]. MRI is increasingly being used, with improved visualization of carcinoid tumors on T2-weighted images [8]. Additional information can be gained from nuclear medicine imaging scans, specifically utilizing Technetium-99m isotopes, as was done with our patient [9]. Finally, if carcinoid is diagnosed postoperatively on histopathology, workup for a primary gastrointestinal site should continue with upper and lower gastrointestinal endoscopy, if these were not performed preoperatively.

The differentiation between primary and secondary NETs of the liver is not possible by histology alone, although a centrally located solitary tumor may suggest a primary [3]. Additionally, some epithelial tumors (e.g. well-differentiated hepatocellular carcinoma, adenocarcinomas and other neoplasms) may exhibit a NET-like morphology. In such cases, immunohistochemical staining for neuroendocrine markers (e.g. chromogranin, synaptophysin, CD56) should be performed to establish the cell of origin. However, it should be noted that most laboratories, including our own, use chromogranin $A$ monoclonal antibody to stain for NETs, therefore NETs expressing chromogranin $B$ may be non-reactive with this antibody, as was the case with our specimen.

All neuroendocrine tumors have malignant potential. As such, some authors recommend using the terms "lowgrade neuroendocrine tumor," "well-differentiated neuroendocrine tumor," "well-differentiated endocrine tumor" or "grade I neuroendocrine carcinoma" instead of "carcinoid tumor" to emphasize their biologic behavior. The value of the term "neuroendocrine tumor" reflects a particular phenotype that may respond to specific targeted therapies [10].

Despite the classic low-grade cytoarchitectural morphology present in this patient's tumor, its large size $(5.2 \mathrm{~cm}$ in greatest dimension) and focally infiltrative border are worrisome. As a general principle, NETs smaller than 1.0 $\mathrm{cm}$, in any anatomic location, usually behave in an indolent fashion with only rare recurrences or distant spread while those larger than $2.0 \mathrm{~cm}$ are usually more aggressive [11]. However, this parameter may not be as important in primary hepatic NETs when it is noted that some of the reported cases have tumors ranging in size from 3.0-16 $\mathrm{cm}$ and six of eight patients have remained disease-free after follow-up of more than three years [3]. On the other hand, there are reported cases with sizes ranging from $8.2-26 \mathrm{~cm}$ where three of five patients died as early as seven months post-operatively [12]. The large size of the tumors in this particular series was surmised to be the cause of the unfavorable outcome.

After the appropriate workup of a hepatic mass, initial management is surgical resection when possible. Extent of resection is determined by location and size of the tumor(s), with multicentric bilobar disease often precluding resection. When this is the case, alternative therapies include radiofrequency ablation [7], hepatectomy with transplantation [3], selective hepatic artery embolization [13], regional or systemic chemotherapy, and intravenous octreotide infusion for symptomatic relief. The limited experience with this disease entity makes current recommendations of management difficult. Traditional approaches to hepatic tumors are employed at the discretion of the treating surgeons, gastroenterologists, interventional radiologists, and oncologists.

The rigorous follow-up and frequent monitoring of patients with hepatic carcinoid also serves as screening for recurrent disease. Recurrences have been reported as early as one year postoperatively and as late as thirteen years, and can occur in the liver or in regional lymph nodes [1416]. Distant metastasis without primary hepatic recurrence has not been reported.

\section{Conclusion}

Carcinoid tumors involving the liver are common, but primary hepatic carcinoid tumors are rare. Classification as a primary hepatic tumor requires extensive workup and prolonged follow-up. Regardless of their size, location, and degree of differentiation, NETs have an inherent malignant potential that must be recognized. Management remains surgical resection, with several alternative options available for non-resectable tumors and severe symptoms. The risk of recurrence of primary hepatic carcinoid tumors after resection remains unknown.

\section{Competing interests}

The authors declare that they have no competing interests.

\section{Authors' contributions}

GS drafted the case presentation and literature review sections of this manuscript. AC and HG reviewed the specimens and drafted the review of the pathological findings associated with this disease entity. FA and JO were the primary physicians diagnosing, treating, and currently following the referenced patient.

\section{Acknowledgements}

Written informed consent was obtained from the patient for publication of this case report and the accompanying images. A copy of the written consent is available for review by the Editor-in-Chief of this journal. 


\section{References}

I. Caplin ME, Buscombe JR, Hilson AJ, Jones AL, Watkinson AF, Burroughs AK: Carcinoid tumor. Lancet 1998, 352:799-805.

2. Evers BD: Small Intestine. In Sabiston Textbook of Surgery 17th edition. Edited by: Townsend CM, Beauchamp RD, Evers BM, Mattox KL. Philadelphia: Elsevier Saunders Inc.; 2004:1359-1362.

3. Fenwick SW, Wyatt JI, Toogood GJ, Lodge JP: Hepatic resection and transplantation for primary carcinoid tumors of the liver. Ann Surg 2004, 239(2):210-219.

4. Mehta DC, Warner RR, Parnes I, Weiss M: An I8-year follow-up of primary hepatic carcinoid with carcinoid syndrome. J Clin Gastroenterol 1996, 23(1):60-62.

5. Tohyama T, Matsui K, Kitagawa K: Primary hepatic carcinoid tumor with carcinoid syndrome and carcinoid heart disease: a case report of a patient on long-term follow-up. Intern Med 2005, 44(9):958-962.

6. Shah NA, Urusova IA, D'Agnolo A, Colquhoun SD, Rosenbloom BE, Vener SL, Geller SA, Yiunes M, Lechago J, Heaney AP: Primary hepatic carcinoid tumor presenting as Cushing's syndrome. J Endocrinol Invest 2007, 30(4):327-333.

7. Komatsuda T, Ishida H, Furukawa K, Miyauchi T, Heianna : Primary carcinoid tumor of the liver: report of a case with an emphasis on contrast-enhanced ultrasonographic findings. J Clin Ultrasound 2005, 33(6):302-304.

8. Fujino K, Koito K, Sano S, Takahara T, Nakamura E, Morisaki $Y$, Furuya T, Torigoe T, Ishii Y: A primary hepatic carcinoid tumor: evaluation by computed tomography and magnetic resonance imaging. Radiat Med 1998, I6(5):37I-373.

9. Shih WJ, Samayoa L, Shih GL, Milan P: Primary hepatic carcinoid tumor presenting as a large multicystic lesion of the liver and on Tc-99m RBC abdominal imaging showing photopenic areas. Clin Nucl Med 2005, 30(7):530-531.

10. DeLellis RA, Osamura RY: Neuroendocrine tumors: an overview. Pathology Case Reviews 2006, I I (6):229-234

II. Graeme-Cook F: Neuroendocrine tumors of the GI tract and appendix. In Surgical Pathology of the GI Tract, Liver, Biliary Tract, and Pancreas Ist edition. Edited by: Odze R. Philadelphia: Elsevier Saunders Inc.; 2003:485-486

12. Pilichowska M, Kimura N, Ouchi A, Lin H, Mizuno Y, Nagura H: Primary hepatic carcinoid and neuroendocrine carcinoma: clinicopathological and immunohistochemical study of five cases. Pathol Int 1999, 49(4):318-324.

13. Sano K, Kosuge T, Yamamoto J, Shimada K, Takayama T, Yamsaki S Makuuchi M: Primary hepatic carcinoid tumors confirmed with long-term follow-up after resection. Hepatogastroenterology 1999, 46(28):2547-2550.

14. Abdel Wahab M, Fathy O, Elghwalby N, Sultan A, Mostafa M, El-Baz M, Elsaadany M, Elshobary M, Ezzat F: Primary hepatic carcinoid tumor: one Egyptian center experience. Hepatogastroenterology 2006, 53(67):33-38

15. limuro $Y$, Deguchi $Y$, Ueda $Y$, Tanaka A, Iwasa $Y$, Ishihara M, Mizuta K, Yamamoto Y, Ikai I, Shimahara Y, Yamaoka Y: Primary hepatic carcinoid tumor with metachronous lymph node metastasis after long-term follow up. J Gastroenterol Hepatol 2002, I7(10): III9-1124.

16. Nishimori H, Tsuji K, Miyamoto N, Sakurai Y, Mitsui S, Kang JH, Yoshida M, Nomura M, Fuminori I, Ishiwatari H: Recurrence of primary hepatic carcinoid tumor in the remnant liver 13 yr after resection. Int J Gastrointest Cancer 2005, 35(2): |47-I5I.
Publish with Biomed Central and every scientist can read your work free of charge

"BioMed Central will be the most significant development for disseminating the results of biomedical research in our lifetime. "

Sir Paul Nurse, Cancer Research UK

Your research papers will be:

- available free of charge to the entire biomedical community

- peer reviewed and published immediately upon acceptance

- cited in PubMed and archived on PubMed Central

- yours - you keep the copyright

Submit your manuscript here:

http://www.biomedcentral.com/info/publishing_adv.asp
BioMedcentral 